\title{
Changes in nuclear structure along the Mn isotopic chain studied via charge radii
}

H. Heylen, ${ }^{1,}{ }^{*}$ C. Babcock,,${ }^{2,3, \dagger}$ R. Beerwerth, ${ }^{4,5}$ J. Billowes, ${ }^{6}$ M. L. Bissell, ${ }^{1,6}$ K. Blaum, ${ }^{7}$ J. Bonnard, ${ }^{8}$ P. Campbell, ${ }^{6}$ B. Cheal, ${ }^{2}$ T. Day Goodacre, ${ }^{6,9}$ D. Fedorov, ${ }^{10}$ S. Fritzsche, ${ }^{4,5}$ R. F. Garcia Ruiz, ${ }^{1}$ W. Geithner, ${ }^{11}$ Ch. Geppert, ${ }^{12,13}$ W. Gins, ${ }^{1}$ L. K. Grob, ${ }^{9,13}$ M. Kowalska, ${ }^{9}$ K. Kreim,${ }^{7}$ S. M. Lenzi, ${ }^{14}$ I. D. Moore, ${ }^{15,16}$ B. Maass, ${ }^{13}$ S. Malbrunot-Ettenauer, ${ }^{9}$ B. Marsh, ${ }^{9}$ R. Neugart, ${ }^{7,12}$ G. Neyens, ${ }^{1}$ W. Nörtershäuser, ${ }^{13}$ T. Otsuka, ${ }^{17}$ J. Papuga, ${ }^{1}$ R. Rossel, ${ }^{9}$ S. Rothe, ${ }^{9}$ R. Sánchez, ${ }^{11}$ Y. Tsunoda, ${ }^{18}$ C. Wraith, ${ }^{2}$ L. Xie, ${ }^{6}$ X. F. Yang, ${ }^{1}$ and D. T. Yordanov ${ }^{7, \ddagger}$

${ }^{1}$ KU Leuven, Instituut voor Kern- en Stralingsfysica, 3001 Leuven, Belgium

${ }^{2}$ Oliver Lodge Laboratory, Oxford Street, University of Liverpool, L69 7ZE, United Kingdom

${ }^{3}$ ISOLDE, Experimental Physics Department, CERN, CH-1211 Geneva 23, Switzerland

${ }^{4}$ Helmholtz Institute Jena, Fröbelstieg 3, 07743 Jena, Germany

${ }^{5}$ Theoretisch-Physikalisches Institut, Friedrich-Schiller-Universität Jena, 07743 Jena, Germany

${ }^{6}$ School of Physics and Astronomy, University of Manchester, Manchester M13 9PL, United Kingdom

${ }^{7}$ Max-Planck-Institut für Kernphysik, D-69117 Heidelberg, Germany

${ }^{8}$ Istituto Nazionale di Fisica Nucleare, Sezione di Padova, 35131 Padova, Italy

${ }^{9}$ ISOLDE, Physics Department, CERN, CH-1211 Geneva 23, Switzerland

${ }^{10}$ Petersburg Nuclear Physics Institute, 188350 Gatchina, Russia

${ }^{11}$ GSI Helmholtzzentrum für Schwerionenforschung GmbH, D-64291 Darmstadt, Germany

${ }^{12}$ Johannes Gutenberg-Universität Mainz, Institut für Kernchemie, D-55128, Germany

${ }^{13}$ Institut für Kernphysik, TU Darmstadt, D-64289 Darmstadt, Germany

${ }^{14}$ Dipartimento di Fisica e Astronomia dell'Università and INFN, Sezione di Padova, Padova, Italy

${ }^{15}$ Department of Physics, University of Jyväskylä, PB 35 (YFL) Jyväskylä, Finland

${ }^{16}$ Helsinki Institute of Physics, FI-00014, University of Helsinki, Finland

${ }^{17}$ Department of Physics, University of Tokyo, Hongo, Bunkyo-ku, Tokyo 113-0033, Japan

${ }^{18}$ Center for Nuclear Study, University of Tokyo, Hongo, Bunkyo-ku, Tokyo 113-0033, Japan

(Received 16 September 2016; published 28 November 2016)

\begin{abstract}
The hyperfine spectra of ${ }^{51,53-64} \mathrm{Mn}$ were measured in two experimental runs using collinear laser spectroscopy at ISOLDE, CERN. Laser spectroscopy was performed on the atomic $3 d^{5} 4 s^{2}{ }^{6} S_{5 / 2} \rightarrow 3 d^{5} 4 s 4 p^{6} P_{3 / 2}$ and ionic $3 d^{5} 4 s^{5} S_{2} \rightarrow 3 d^{5} 4 p^{5} P_{3}$ transitions, yielding two sets of isotope shifts. The mass and field shift factors for both transitions have been calculated in the multiconfiguration Dirac-Fock framework and were combined with a King plot analysis in order to obtain a consistent set of mean-square charge radii which, together with earlier work on neutron-deficient $\mathrm{Mn}$, allow the study of nuclear structure changes from $N=25$ across $N=28$ up to $N=39$. A clear development of deformation is observed towards $N=40$, confirming the conclusions of the nuclear moments studies. From a Monte Carlo shell-model study of the shape in the Mn isotopic chain, it is suggested that the observed development of deformation is not only due to an increase in static prolate deformation but also due to shape fluctuations and triaxiality. The changes in mean-square charge radii are well reproduced using the Duflo-Zuker formula except in the case of large deformation.
\end{abstract}

DOI: 10.1103/PhysRevC.94.054321

\section{INTRODUCTION}

The structure of exotic nuclei exhibits many peculiarities such as halo nuclei, islands of inversion, and shape coexistence. Laser spectroscopy has played a decisive role in studying these phenomena as it enables measurements of spins, nuclear

\footnotetext{
*hanne.heylen@fys.kuleuven.be

†cbabcock@triumf.ca

${ }^{\ddagger}$ Present address: Institut de Physique Nucléaire, CNRS-IN2P3, Univ. Paris-Sud, Université Paris-Saclay, 91406 Orsay, France.
}

Published by the American Physical Society under the terms of the Creative Commons Attribution 3.0 License. Further distribution of this work must maintain attribution to the author(s) and the published article's title, journal citation, and DOI. moments, and changes in mean-square charge radii from the valley of stability to exotic regions of the nuclear chart [1]. Due to the complementarity of the measured ground-state properties, a comprehensive description of nuclear structure can be established, for example, the evolution of both singleparticle and collective aspects as a function of nucleon number [2,3]. Mean-square charge radii in particular have been systematically measured across the nuclear chart. They are sensitive to the shape and size of the nucleus and can also be extracted for even-even nuclei with $I=0$, for which the magnetic moments and spectroscopic quadrupole moments are zero.

The region between the proton-magic $\mathrm{Ca}(Z=20)$ and Ni $(Z=28)$ isotopes has attracted much attention due to its rich variety in nuclear structure. Around calcium, early measurements revealed a remarkable behavior of the charge radii between $N=20$ and $N=28$ [4-7]. Furthermore, the 
appearance of new magic numbers at $N=32$ and $N=34$ was investigated [8-12]. Adding a few protons towards the nickel isotopic chain and a few neutrons towards $N=40$, a rapid development of collectivity is observed, bearing close resemblance to the island of inversion around $N=20[13,14]$. Although nuclear moments and mean-square charge radii have been essential in the interpretation of the nuclear structure around $N=20$ [15-18], so far the experimental knowledge of these observables around $N=40$ is scarce. Their measurements therefore provide necessary and complementary information.

With 25 protons, the manganese isotopes lie centrally between the $\mathrm{Ca}$ and $\mathrm{Ni}$ isotopic chains. The nuclear moments and mean-square charge radii of $\mathrm{Mn}$ between $N=25$ and $N=31$ were previously studied at the IGISOL facility, JYFL, Jyväskylä [19]. These measurements have now been extended to $N=39$ in two collinear laser spectroscopy experiments at ISOLDE, CERN [20-22]. With the ground-state properties of Mn known from $N=25$ across $N=28$ up to $N=39$, a detailed picture of the changing nuclear structure emerges. The mean-square charge radii and quadrupole moments show a clear kink at $N=28$ associated with a neutron shell closure, while the effect of $N=28$ on the two-neutron separation energies is not immediately apparent [19]. The steeply increasing slope of the radii between $N=28$ and $N=32$ is found to be common to the isotopic chains in this region, indicating an almost $Z$-independent core-polarization by neutrons filling the upper $p f$ shell [23]. Going even more neutron-rich, the Mn magnetic and quadrupole moments show evidence of increased collectivity towards $N=40$ [20-22]. Comparisons with large-scale shell-model calculations have illustrated the importance of particle-hole excitations across $N=40$ and $Z=28$ for the description of the nuclear structure approaching $N=40$.

In this article, the mean-square charge radii of ${ }^{51,53-64} \mathrm{Mn}$ measured in the two previously mentioned ISOLDE experiments are presented. $\mathrm{Mn}$ is to date the first isotopic chain below $Z=28$ for which the ground-state properties beyond $N=28$ can be systematically studied for such a long sequence of isotopes.

\section{EXPERIMENTAL METHOD}

The experimental campaign was performed at the ISOLDE radioactive ion beam facility in CERN where exotic manganese isotopes were produced via the bombardment of a thick uranium carbide target with $1.4-\mathrm{GeV}$ protons. Element selective ionization was achieved using the resonance ionization laser ion source (RILIS) [24]. After mass separation in the HRS, the isotopically pure ion beam was cooled and bunched in the gas-filled RFQ ISCOOL [25] and reaccelerated to, respectively, 40 and $30 \mathrm{keV}$, in the first and second experiment. The bunched beam was then guided to the dedicated collinear laser spectroscopy beam line COLLAPS [26-28] where it was overlapped with a copropagating narrow-band laser beam. A Doppler-tuning potential was applied to scan the laser frequency across the hyperfine structure in the reference frame of the ions or atoms. The fluorescence light from the decay following the resonant laser-induced excitations between the hyperfine levels was detected using sensitive photomultiplier tubes placed perpendicular to the beam line.

In a first experiment, laser spectroscopy was performed on an atomic transition from the $3 d^{5} 4 s^{2}{ }^{6} S_{5 / 2}$ ground state to the $3 d^{5} 4 s 4 p{ }^{6} P_{3 / 2}$ excited state at a wavelength of $280.1907 \mathrm{~nm}$ (in vacuum). Hyperfine spectra of ${ }^{51,53-64} \mathrm{Mn}$ were obtained. The details of the experiment and data analysis are described in Refs. [20,21]. To improve the sensitivity to quadrupole moments, a second experiment was performed on an ionic transition at $295.0066 \mathrm{~nm}$ (in vacuum) from the $3 d^{5} 4 s{ }^{5} S_{2}$ metastable state at $9472.97 \mathrm{~cm}^{-1}$ to the $3 d^{5} 4 p{ }^{5} P_{3}$ state at $43370.51 \mathrm{~cm}^{-1}$. The population of the ionic metastable state was enhanced using the technique of optical pumping in the ISCOOL cooler/buncher, as outlined in Ref. [22]. In this second experiment, only hyperfine spectra of the odd-even ${ }^{53-63} \mathrm{Mn}$ isotopes were measured.

\section{RESULTS}

Typical hyperfine spectra measured on the atomic and ionic transition are shown in Figs. 1 and 2, respectively. The centroid frequency $v_{0}^{A}$ for each spectrum is indicated with a vertical dashed line. The isotope shifts relative to ${ }^{55} \mathrm{Mn}$

$$
\delta v^{55, A}=v_{0}^{A}-v_{0}^{55}
$$

extracted from these hyperfine spectra are presented in Table I; the hyperfine $A$ and $B$ parameters were reported earlier in Refs. [20-22]. Statistical errors obtained in the fit procedure are indicated with round brackets while a systematic error due to $\mathrm{a} \pm 15 \mathrm{~V}$ uncertainty in the acceleration voltage calibration is assumed for both experiments, indicated in square brackets. For completeness, also the isotope shifts measured previously at the IGISOL facility JYFL in Jyväskylä [19] using the same ionic transition are reported in Table I.

The measured isotope shifts can be related to the changes in mean-square charge radii $\delta\left\langle r^{2}\right\rangle$ using (see, e.g., Ref. [3])

$$
\delta v_{j}^{55, A}=M_{j} \frac{m_{A}-m_{55}}{m_{A} m_{55}}+F_{j} \delta\left\langle r^{2}\right\rangle^{55, A} .
$$

Here $m_{A}$ is the mass of an isotope $A$ and $M_{j}$ and $F_{j}$ are the mass and field shift parameters specific to the electronic transition $j$. The first term (mass shift) is due to the recoil kinetic energy of a nucleus with finite mass while the second term (field shift) originates from the changes in nuclear charge distribution and hence contains the charge radius dependence. To reliably extract the changes in mean-square charge radii, an accurate knowledge of both electronic parameters is required. In first-order perturbation theory, these parameters are constant along an isotopic chain but they need to be determined for each electronic transition separately.

\section{A. Electronic factor calculations}

For Mn, the mass and field shift factors cannot be determined by a direct comparison between the measured isotope shifts and the independently known charge radii since the absolute charge radius of only one isotope is known from nonoptical methods. Therefore, one has to rely on theoretical 




FIG. 1. Hyperfine spectra of ${ }^{51,53-64} \mathrm{Mn}$ measured on the atomic ${ }^{6} S_{5 / 2} \rightarrow{ }^{6} P_{3 / 2}$ transition. The centroid for each spectrum is indicated with a vertical dashed line. For ${ }^{58,60,62} \mathrm{Mn}$ only the centroid frequency of the ground state is indicated (the isomer shift is too small to be visible on this scale).

calculations to obtain the electronic $M$ and $F$ factors, using, e.g., the multiconfiguration Dirac-Fock (MCDF) method [29].

The MCDF method, implemented in the GRASP2K code [30], is applied to generate the orbitals for the atomic $3 d^{5} 4 s^{2}{ }^{6} S_{5 / 2}$ and $3 d^{5} 4 s 4 p^{6} P_{3 / 2}$ configurations as well as the ionic $3 d^{5} 4 s^{5} S_{2}$ and $3 d^{5} 4 p^{5} P_{3}$ levels. In a subsequent step, the Breit interaction is included by first-order perturbation theory



FIG. 2. Hyperfine spectra of the odd-even ${ }^{53-63} \mathrm{Mn}$ isotopes measured on the ionic ${ }^{5} S_{2} \rightarrow{ }^{5} P_{3}$ transition. The centroid frequency for each spectrum is indicated with a vertical dashed line. Due to the limited yield at ${ }^{63} \mathrm{Mn}$ only the last three multiplets in the hyperfine spectrum were measured, sufficient to extract the centroid and hyperfine parameters.

in the configuration-interaction method [31]. The resulting wave functions are then used to compute the mass shift parameters by utilizing the RIS3 module [32] and the field shift parameter is computed using the RATIP tools [33]. This distinguishes the current results from the previous computations on the same ionic transition reported in Ref. [19], where the relativistic correction to the mass shift operator was neglected. However, these corrections do not exceed the uncertainty due to the complex electronic structure. The open $3 d$ shell makes theoretical calculations on atomic and singly ionized manganese very challenging and extensive in terms of required computational power.

In the MCDF approach, the wave function is represented as a superposition of configuration state functions (CSFs). Each CSF represents a specific electronic configuration and the choice of this basis set is crucial for the quality of the obtained results. In the lowest-order approximation, the model space consists of the $3 d, 4 s$, and $4 p$ valence shells. This space is then extended by adding a set of correlation orbitals $n l$, which are populated by virtual excitations from the reference configurations. Due to computational restrictions, the correlation orbitals are limited to orbitals with angular momentum up to $f$. In subsequent steps, the model space is systematically extended by further layers with a new principal quantum number $n$. The virtue of this model is that it allows convergence of the desired quantities to be monitored as the model space is expanded. For all computations performed in this work, single and double excitations from the valence 
TABLE I. Mn isotope shifts relative to ${ }^{55} \mathrm{Mn}$ measured on the atomic $3 d^{5} 4 s^{2}{ }^{6} S_{5 / 2} \rightarrow 3 d^{5} 4 s 4 p{ }^{6} P_{3 / 2}$ transition and the ionic $3 d^{5} 4 s{ }^{5} S_{2} \rightarrow 3 d^{5} 4 p{ }^{5} P_{3}$ transition. Additional to the statistical uncertainty in round brackets, the systematic uncertainty on the isotope shifts due to a $\pm 15 \mathrm{~V}$ uncertainty on the calibration of the acceleration voltage is indicated in square brackets.

\begin{tabular}{|c|c|c|c|}
\hline$A$ & $N$ & $\delta v_{\text {atom }}^{55, A}(\mathrm{MHz})$ & $\delta v_{\text {ion }}^{55, A}(\mathrm{MHz})$ \\
\hline $50 g$ & 25 & & $-1573(2)[27]^{\mathrm{a}}$ \\
\hline $50 m$ & 25 & & $-1514(8)[27]^{\mathrm{a}}$ \\
\hline 51 & 26 & $-1698(7)[20]$ & $-1201(26)[21]^{\mathrm{a}}$ \\
\hline $52 g$ & 27 & & $-745(7)[16]^{\mathrm{a}}$ \\
\hline $52 m$ & 27 & & $-782(3)[16]^{\mathrm{a}}$ \\
\hline \multirow[t]{2}{*}{53} & 28 & $-669(6)[9]$ & $-418(3)[10]$ \\
\hline & & & $-418(2)[10]^{\mathrm{a}}$ \\
\hline 54 & 29 & $-307(6)[5]$ & $-192(4)[5]^{\mathrm{a}}$ \\
\hline 55 & 30 & 0 & 0 \\
\hline 56 & 31 & $340(7)[5]$ & $240(6)[5]^{\mathrm{a}}$ \\
\hline 57 & 32 & $655(7)[9]$ & $422(5)[10]$ \\
\hline $58 g$ & 33 & $983(4)[13]$ & \\
\hline $58 m$ & 33 & $990(4)[13]$ & \\
\hline 59 & 34 & $1284(5)[17]$ & $863(11)[19]$ \\
\hline $60 g$ & 35 & $1607(10)[21]$ & \\
\hline $60 m$ & 35 & $1594(4)[21]$ & \\
\hline 61 & 36 & $1843(7)[25]$ & $1215(2)[27]$ \\
\hline $62 g$ & 37 & $2099(8)[28]$ & \\
\hline $62 m$ & 37 & $2088(5)[28]$ & \\
\hline 63 & 38 & $2355(6)[32]$ & $1542(5)[35]$ \\
\hline 64 & 39 & $2566(6)[35]$ & \\
\hline
\end{tabular}

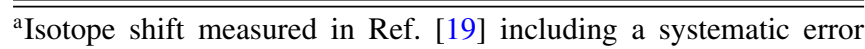
arising from $\mathrm{a} \pm 15 \mathrm{~V}$ uncertainty of the calibration in the acceleration voltage.

shells, as well as single excitations from all core shells, are taken into account. All calculations are performed with common core orbitals, while the correlation orbitals are separately optimized for the upper and lower levels.

For the atomic calculations, the reference configurations $3 d^{5} 4\left(s^{2}+p^{2}\right)$ for the ground-state and the excited-state configurations $3 d^{5} 4 s 4 p$ and $3 d^{6} 4 s$ are used to generate five correlation layers. This choice of the reference configurations yields a well-balanced basis expansion such that the transition energy as well as the $M$ and $F$ parameters converge well.

The computations on the ionic transitions show much more complex electron correlations. It is found that the two levels of interest significantly correlate with configurations where at least one electron is excited to the $4 f$ or $4 d$ shell. A complete treatment of this kind of correlations is not possible due to the extremely large basis expansions. Therefore, an alternative approach is used where the zeroth-order approximation is computed with a space spanned by the $3 d^{5} 4 s, 3 d^{4} 4 p^{2}$, and $3 d^{3} 4 s 4 d^{2}$ configurations for the lower level and $3 d^{5} 4 p$, $3 d^{4} 4 s 4 p$, and $3 d^{3} 4 p 4 d^{2}$ for the upper level. The correlation layers were then generated by using only the $3 d^{5}(4 s+4 p)$ configurations as a reference. This approach yields good convergence for the first four layers; however, convergence fails for the fifth layer, possibly due to neglected electronic correlations.
TABLE II. Overview of the electronic mass and field shift factors for the atomic $3 d^{5} 4 s^{2}{ }^{6} S_{5 / 2} \rightarrow 3 d^{5} 4 s 4 p{ }^{6} P_{3 / 2}$ transition and the ionic $3 d^{5} 4 s^{5} S_{2} \rightarrow 3 d^{5} 4 p^{5} P_{3}$ transition calculated in the multiconfiguration Dirac-Fock framework. Also the atomic factors calculated with respect to (wrt) the ionic factors using the King plot analysis are shown and vice versa. The estimated theoretical uncertainties on the parameters are indicated in square brackets, and where applicable, the fit errors from the King plot are indicated in round brackets.

\begin{tabular}{lccc}
\hline \hline Transition & Method & $M(\mathrm{GHz})$ & $F\left(\mathrm{MHz} / \mathrm{fm}^{2}\right)$ \\
\hline Atom: & MCDF & $\mathbf{+ 1 1 5 8}[81]$ & $\mathbf{- 4 6 5}[70]$ \\
${ }^{6} S_{5 / 2} \rightarrow{ }^{6} P_{3 / 2}$ & King plot wrt & $+1188(108)[65]$ & $-525(39)[93]$ \\
& MCDF 2010 & & \\
Ion: & MCDF 4 & $+875[131]$ & $-546[82]$ \\
${ }^{5} S_{2} \rightarrow{ }^{5} P_{3}$ & MCDF 2010[19] & $+852[60]$ & $-572[86]$ \\
& King plot wrt & $\mathbf{+ 8 1 9}(125)[88]$ & $\mathbf{- 5 0 6}(38)[76]$ \\
& MCDF atom & & \\
\hline \hline
\end{tabular}

An overview of the different electronic factors obtained from the computations described above and in Ref. [19] (labeled MCDF 2010) can be found in Table II and a comparison of the changes in charge radii evaluated with those factors is shown in Fig. 3. There is good agreement between the charge radii obtained with the electronic factors for the atomic transition calculated in this work and the previously published calculations on the ionic transition. The computation on the ionic transition including up to four correlation layers (this work) yields slightly larger differences in charge radii, but the agreement is still better than the uncertainty due to the omitted electronic correlations. Due to the aforementioned convergence problems of the ionic calculations and the limited treatment of electronic correlations, the atomic calculations are considered to be more reliable. Therefore, the atomic results are used in the following King plot analysis.



FIG. 3. Comparison between the changes in mean-square charge radii between $N=28$ and $N=39$ calculated using the mass and field shift factors obtained in three different MCDF calculations. Only the statistical experimental uncertainties are shown. 


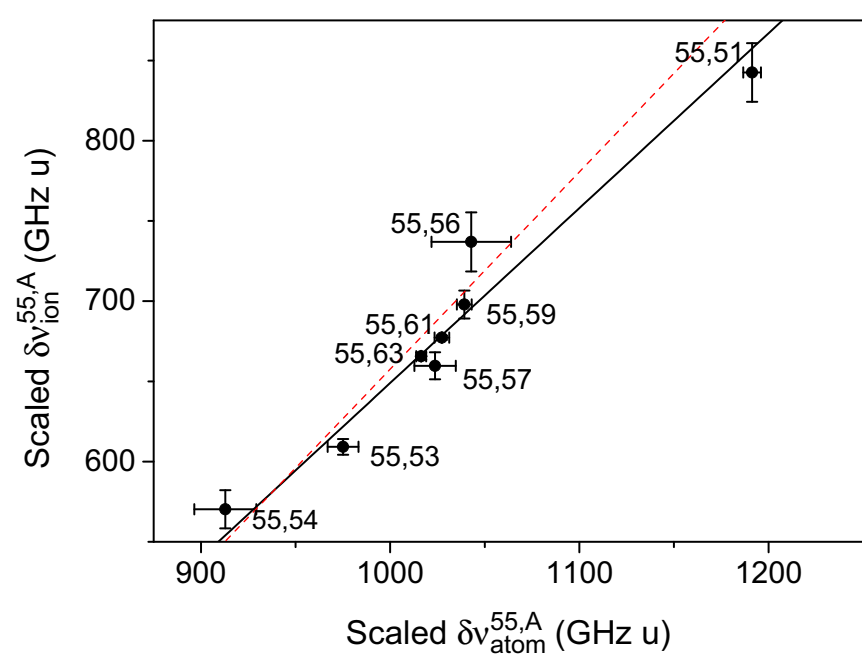

FIG. 4. King plot using the isotope shifts of the atomic and ionic transitions, shown in Table I. The best linear fit (black solid line) is shown together with the linear relation obtained by filling in the atomic MCDF and the ionic MCDF 2010 factors in Eq. (2) (dashed red line).

An additional consistency check of the calculations is performed via the King plot method [34]

$$
\mu^{55, A} \delta v_{\text {ion }}^{55, A}=\frac{F_{\text {ion }}}{F_{\text {atom }}} \mu^{55, A} \delta \nu_{\text {atom }}^{55, A}+M_{\text {ion }}-\frac{F_{\text {ion }}}{F_{\text {atom }}} M_{\text {atom }},
$$

where $\mu^{55, A}=m^{55} m^{A} /\left(m^{A}-m^{55}\right)$ is used as a scaling factor for the isotope shifts. In Fig. 4 the scaled isotope shifts of the ionic transition are plotted against those of the atomic transition. A linear fit then gives the slope $\frac{F_{\text {ion }}}{F_{\text {atom }}}=1.09(8)$ and intercept $M_{\text {ion }}-\frac{F_{\text {ion }}}{F_{\text {atom }}} M_{\text {atom }}=-44(8) \times 10^{4} \mathrm{MHz} \mathrm{u}$ from which the mass and field shift parameters of the ionic transition can be determined from the atomic computation and vice versa (see Table II).

In general, the estimation of the uncertainty on the mass and field shift factor calculations is not straightforward since the main source of error, omitted electronic correlations, is difficult to quantify. Therefore, rather conservative theoretical uncertainties of $15 \%$ are commonly assumed. However, in this study, comparison of the independently computed values of the ionic and atomic transition, correlated to each other using the King plot method permits us to verify these uncertainties. This comparison suggests that an uncertainty of $15 \%$ on $F$ is realistic while a $15 \%$ uncertainty on $M$ seems overestimated. From the agreement of the King plot analysis, an uncertainty of $7 \%$ on $M$ seems to be more appropriate.

\section{B. Changes in mean-square charge radii}

The changes in mean-square charge radii relative to ${ }^{55} \mathrm{Mn}$ are determined using the experimental isotope shifts in Table I and the electronic factors shown in bold in Table II. Because a King plot is used to link the mass and field shift factors of the ionic transition to the atomic transition, the charge radii extracted from the two transitions are consistent with each other, as can be seen in Table III. Nevertheless, since
TABLE III. Changes in mean-square charge radii of ${ }^{50-64} \mathrm{Mn}$ relative to ${ }^{55} \mathrm{Mn}$. These are extracted from the isotope shifts measured on the atomic ${ }^{6} S_{5 / 2} \rightarrow{ }^{6} P_{3 / 2}$ and the ionic ${ }^{5} S_{2} \rightarrow{ }^{5} P_{3}$ transition in combination with the electronic parameters shown in bold in Table II. The experimental errors consist of the statistical uncertainties and the systematic uncertainties due to the uncertainty on the acceleration voltage, shown in round and square brackets, respectively. No theoretical uncertainties due to the uncertainty on the calculated mass and field shift factors are shown, as explained in Sec. III B.

\begin{tabular}{lllc}
\hline \hline$A$ & $N$ & \multicolumn{2}{c}{$\delta\left\langle r^{2}\right\rangle^{55, A}\left(\mathrm{fm}^{2}\right)$} \\
\cline { 2 - 4 } & & \multicolumn{1}{c}{ Atom } & Ion \\
\hline $50 g$ & 25 & & $0.168(4)[53]$ \\
$50 m$ & 25 & & $0.051(16)[53]$ \\
51 & 26 & $0.102(14)[42]$ & $0.065(53)[41]$ \\
$52 g$ & 27 & & $-0.226(14)[31]$ \\
$52 m$ & 27 & & $-0.153(6)[31]$ \\
53 & 28 & $-0.270(12)[20]$ & $-0.285(7)[20]$ \\
54 & 29 & $-0.177(12)[10]$ & $-0.166(8)[10]$ \\
55 & 30 & 0 & 0 \\
56 & 31 & $0.081(15)[10]$ & $0.053(12)[10]$ \\
57 & 32 & $0.185(15)[19]$ & $0.202(11)[19]$ \\
$58 g$ & 33 & $0.234(8)[28]$ & \\
$58 m$ & 33 & $0.220(8)[28]$ & $0.297(21)[37]$ \\
59 & 34 & $0.316(10)[37]$ & \\
$60 g$ & 35 & $0.329(14)[45]$ & $0.504(5)[54]$ \\
$60 m$ & 35 & $0.357(9)[45]$ & \\
61 & 36 & $0.504(15)[53]$ & $0.704(10)[69]$ \\
$62 g$ & 37 & $0.615(18)[61]$ & \\
$62 m$ & 37 & $0.640(9)[61]$ & \\
63 & 38 & $0.706(13)[69]$ & \\
64 & 39 & $0.873(14)[76]$ & \\
\hline \hline
\end{tabular}

the charge radii are obtained by using only two parameters for each transition, the one-by-one agreement between the two transitions provides an independent cross-check of the consistency of the isotope shift measurements.

In Table III no theoretical uncertainties are shown. This is justified because although the theoretical uncertainty on the electronic factors gives a large uncertainty on the exact $\delta\left\langle r^{2}\right\rangle$ value, as illustrated in Fig. 5, it does not alter the discussion in Sec. IV. Indeed, a variation of $M$ and $F$ causes a general pivot about the reference point but does not change the local trends, as illustrated in Fig. 6. Since nuclear structure effects manifest themselves as local irregularities in the course of the charge radii, the interpretation is not affected by the theoretical uncertainty.

\section{DISCUSSION}

\section{A. Mn radii systematics}

In Fig. 5 the mean-square charge radii $\left\langle r^{2}\right\rangle$ of the ${ }^{50-64} \mathrm{Mn}$ isotopes $(N=25-39)$ are plotted along with the charge radii of the neighboring isotopes between ${ }_{19} \mathrm{~K}$ and ${ }_{29} \mathrm{Cu}$. For $\mathrm{Mn}$ the results from the atomic transition are shown except for ${ }^{50,51,52,54,56} \mathrm{Mn}$ for which only ionic data is available. The $\left\langle r^{2}\right\rangle$ values are obtained with the reference radii of Fricke and 


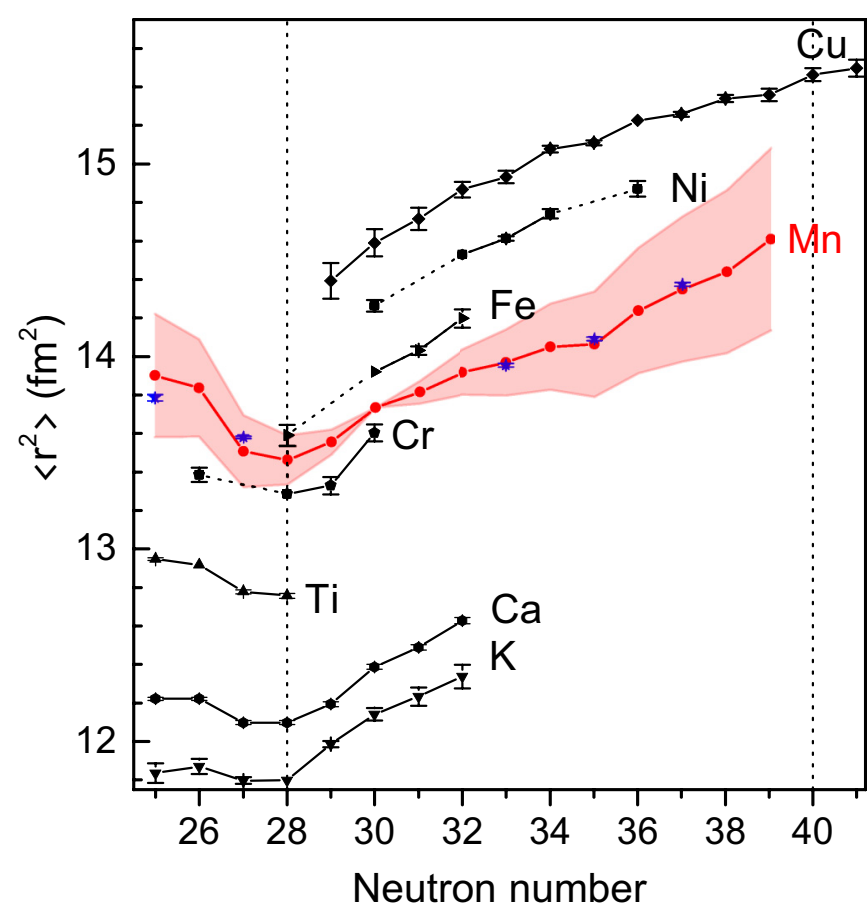

FIG. 5. The experimental mean-square charge radii known in the Mn region between $N=25$ and $N=39$. The Mn isotopes are highlighted in red with the isomers in ${ }^{50,52,58,60,64} \mathrm{Mn}$ indicated by a blue star. The shaded area represents the theoretical uncertainty assuming a $15 \%$ uncertainty on $F$ and $7 \%$ on $M$.

Heilig [35] and the published changes in mean-square charge radii [12,23,36-39].

A first observation is that the mean-square charge radii of the $4^{+}$isomers in neutron-rich ${ }^{58,60,62} \mathrm{Mn}$ are very close to the $1^{+}$ground-state radii, as indicated by the blue stars in Fig. 5. This suggests similar degrees of deformation and no sizable shape coexistence. Although the absolute difference in charge radii of the two states is small, the charge radius of the isomer in ${ }^{58} \mathrm{Mn}$ is slightly smaller than that of the ground state, while it is slightly larger in ${ }^{60} \mathrm{Mn}$ and ${ }^{62} \mathrm{Mn}$. Note that the difference in isomer and ground-state charge radii is much larger on the neutron-deficient side. In the self-conjugate $N=Z=25$ nucleus ${ }^{50} \mathrm{Mn}$ this can be understood in terms of the difference in isospin ( $T=0$ and $T=1)$, as was discussed previously for ${ }_{19}^{38} \mathrm{~K}_{19}$ [40].

A strong effect of the $N=28$ shell closure is seen as a local minimum in the mean-square charge radii of all isotopic chains. Moreover, the increase in charge radii beyond $N=28$ is strikingly independent of $Z$, as pointed out previously in a study of the neutron-rich ${ }_{19} \mathrm{~K}$ isotopes [23]. In Fig. 6, the changes in mean-square charge radii of Mn determined in this work are plotted together with those of ${ }_{26} \mathrm{Fe}$ [37], ${ }_{28} \mathrm{Ni}$ [38], and ${ }_{29} \mathrm{Cu}$ [39] relative to $N=30$. On first sight, it might seem that the Mn radii do not follow the regional trend, but one has to remember that the theoretical uncertainty on the radii due to the uncertainty on the calculated mass and field shift factors allows for a pivot of the slope within the shaded area. Indeed, when for example the mass shift factor $M_{\text {atom }}$ is increased by $5 \%$, well within the quoted uncertainty, the mean-square

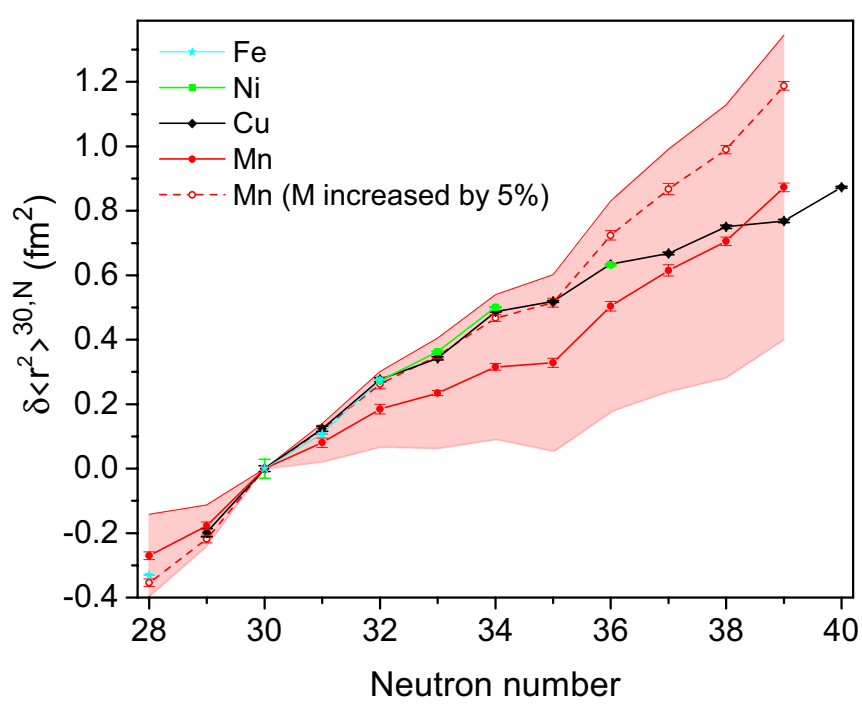

FIG. 6. Changes in mean-square charge radii of $\mathrm{Mn}, \mathrm{Fe}, \mathrm{Ni}$, and $\mathrm{Cu}$ with respect to $N=30$. The shaded area represents the theoretical uncertainty on the Mn radii assuming a $15 \%$ uncertainty on $F$ and $7 \%$ on $M$. To illustrate the meaning of this uncertainty, also the Mn mean-square charge radii in which the mass shift factor $M$ is increased by $5 \%$ are shown.

charge radii of $\mathrm{Mn}$ are found to agree almost perfectly with those of the neighboring isotopic chains, as illustrated by the dashed line and open symbols in Fig. 6. By this depiction, it additionally becomes clear that the apparent dip at $N=35$, which seems anomalous at first, is rather due to a combination of odd-even staggering and a sudden increase in charge radii at $N=36$, as will be discussed next.

Whereas in $\mathrm{Cu}$ the mean-square charge radii exhibit a weakly parabolic behavior between $N=28$ and $N=40$ [39], in $\mathrm{Mn}$ a rapid increase in charge radii is observed from $N=36$ onwards. This observation is consistent with the increase in the spectroscopic quadrupole moments [22] and the behavior of the magnetic moments [20,21], interpreted as arising from particle-hole excitations across $Z=28$ and $N=40$. From $N=36$ onwards, these magnetic and quadrupole moments can be described by large-scale shell-model calculations only if excitations of neutrons from the $p f$ shell into the $g_{9 / 2}$ and $d_{5 / 2}$ orbits are included, as well as proton excitations across $Z=28$.

A good reproduction of the experimental magnetic and quadrupole moments is achieved in the Monte Carlo shell model (MCSM) framework using the modified A3DA interaction [41,42], which includes the full $p f$ shell and $g_{9 / 2} d_{5 / 2}$ orbitals in the model space for both protons and neutrons [22]. This gives confidence in the predictive power of these calculations to discuss the shape of the Mn ground states. A constrained Hartree-Fock calculation using this A3DA shell-model Hamiltonian provides the potential energy surface on which the distribution of the MCSM basis states (deformed Slater determinants) as a function of the intrinsic quadrupole moments $Q_{0} \propto\left\langle 2 z^{2}-x^{2}-y^{2}\right\rangle$ and $Q_{2} \propto\left\langle x^{2}-y^{2}\right\rangle$ is plotted. Figure 7 presents these potential energy surfaces for the ground states of the odd-even ${ }^{53-65} \mathrm{Mn}$ isotopes, showing the 

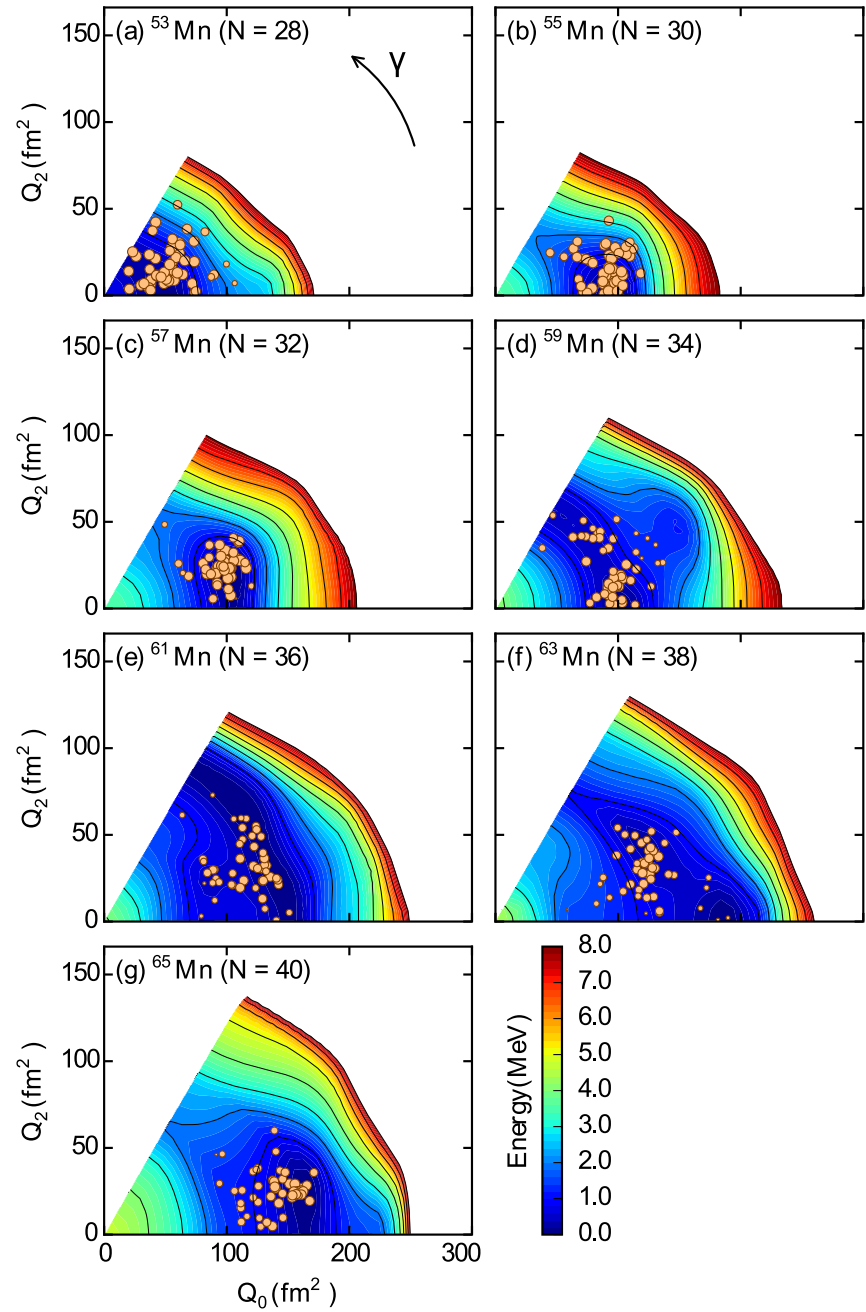

FIG. 7. Potential energy surfaces of odd-even ${ }^{53-63} \mathrm{Mn}$ isotopes, coordinated by $Q_{0}$ and $Q_{2}$ (or alternatively $\gamma$ ranging between $0^{\circ}$ and $60^{\circ}$ ). The distribution of the MCSM basis states is depicted by the circles. The locations of the circles indicate the intrinsic shapes of the MCSM basis states and the sizes denote their importance in the total wave function; see Ref. [41] for more information. The energy scale is relative to the minimum of each potential energy surface, and the contour plots are shown up to a cutoff energy of $8 \mathrm{MeV}$.

evolution of intrinsic shape between $N=28$ and $N=40$. At the $N=28$ shell closure the distribution of intrinsic shapes is centered around the spherical minimum. This changes towards moderately deformed prolate structures between $N=30$ and 34 , then an additional increase in prolate deformation is seen in $N=36$ and 38 , and a maximum is reached at $N=40$. However, a picture in which only axial symmetric deformation is considered is too simple since from $N=34$ onwards a triaxial minimum appears and becomes dominant in $N=36,38$ and $N=40$. Moreover, the observed spread in the distribution of intrinsic shapes points to considerable shape fluctuations in the ground states of the neutron-rich $\mathrm{Mn}$ isotopes. Such shape fluctuations were already suggested to be important in the low-energy structure of the neutron-rich ${ }_{24} \mathrm{Cr}$ isotopes from a constrained Hartree-Fock-Bogoliubov plus local quasiparticle random-phase approximation study [43].
The sudden increase in experimental charge radii is in accordance with the predicted change in nuclear shape at $N=36$ although these calculations indicate that the change in the radii cannot be solely attributed to a change in static prolate deformation.

\section{B. Charge radii and masses}

In this section, the connection between the trend in meansquare charge radii and the masses of the $\mathrm{Mn}$ isotopes is examined. Across the nuclear chart, trends in charge radii are in general closely reflected in the course of the two-neutron separation energies, with clear examples in the $A \sim 100$ and rare-earth regions $[44,45]$. However, this correspondence is not always straightforward, as shown in [19] for the light mass region. For example, the prominent $N=28$ shell closure effect in the Mn charge radii is almost not visible in the two-neutron separation energies. Such discrepancies are not unexpected, since the charge radii and binding energies reflect different aspects of the nuclear wave function. In the context of shape coexistence, for example, it becomes clear that states with very different quantum-mechanical structure can be almost degenerate in energy; thus a huge effect in nuclear size can be accompanied by a small change in nuclear binding. In particular, for the Mn isotopes the $Z \approx N \approx 25$ (also known as the Wigner effect [46]) plays an important role in modifying the trend of the two-neutron separation energies close to $N=28$. Furthermore, a change of ground-state structure when crossing $N=28$ might also bring an additional energy contribution to the trend close to the semimagic ${ }^{53} \mathrm{Mn}_{28}$. Indeed, the spin changes from $5 / 2^{-}$to $7 / 2^{-}$and back to $5 / 2^{-}$in ${ }^{51,53,55} \mathrm{Mn}$, along with a significant increase of their quadrupole moments going from $Q_{s}\left({ }^{53} \mathrm{Mn}\right)=+0.16(3) \mathrm{fm}^{2}$ at the shell closure to $Q_{s}=+0.42(7) \mathrm{fm}^{2}$ and $Q_{s}=+0.33(1) \mathrm{fm}^{2}$ in ${ }^{51,55} \mathrm{Mn}$, respectively $[19,22]$.

Precise mass measurements of the neutron-rich Mn were presented by Naimi et al. [48] noting that it was unclear whether the observed $4^{+}$state in ${ }^{62} \mathrm{Mn}$ was the ground or isomeric state, with a tentative assignment to the isomer. In the mean time, a Coulomb excitation experiment has been performed in which the $4^{+}$state was proposed to lie $346 \mathrm{keV}$ above the $1^{+}$ground state [49]. With this new information, the ground-state two-neutron separation energies can be recalculated, as shown in Fig. 8. The inset highlights the difference between the original (dashed line) and recalculated (solid line) values. While in the original publication a sudden increase in two-neutron separation energies at $N=38$ was observed using the measured ${ }^{62} \mathrm{Mn}$ mass, this effect is washed out using the recalculated ground-state mass. Instead, the two-neutron separation energies gradually curve up towards $N=40$, which would rather be linked to a gradual onset of collectivity [50]. So although the onset of deformation seems to occur suddenly at $N=36$ in the charge radii, this is accompanied by a smooth change of the ground-state binding energies.

Similar to Mn, a smooth upwards curvature is also seen in the ${ }_{26} \mathrm{Fe}$ isotopic chain while an abrupt discontinuity at $N=36$ is observed in the AME2012 evaluation of ${ }_{24} \mathrm{Cr}$ isotopes. However, new measurements show a much smoother 


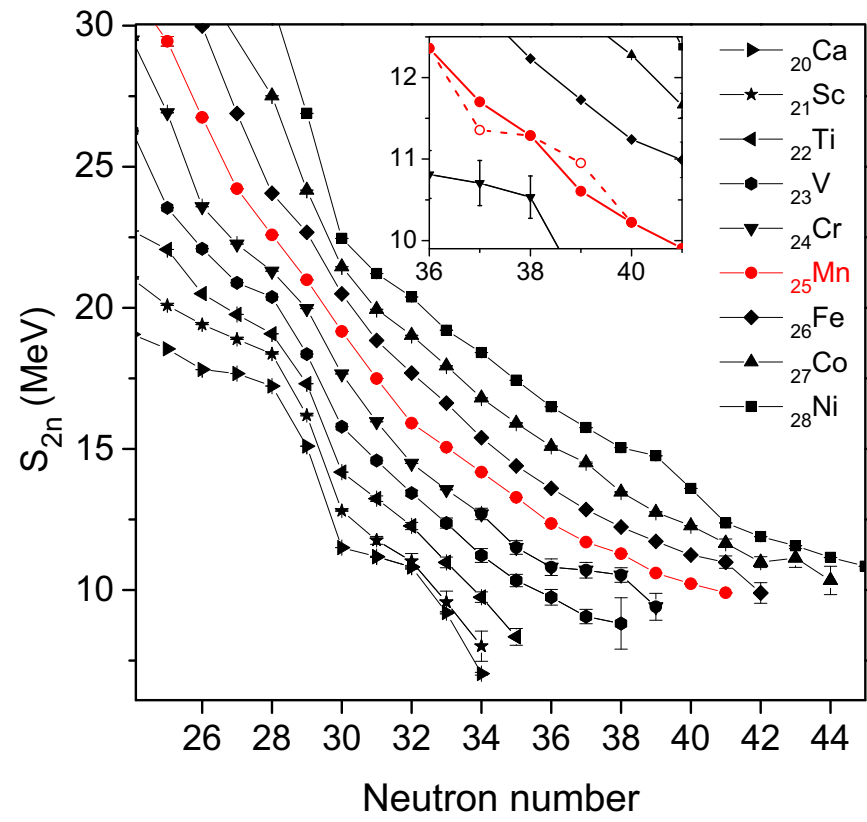

FIG. 8. Two-neutron separation energies plotted from ${ }_{20} \mathrm{Ca}$ to ${ }_{28} \mathrm{Ni}$. Data taken from the AME2012 atomic-mass evaluation [47] complemented with data from Refs. $[10,48]$. The ${ }^{62} \mathrm{Mn}$ binding energy is recalculated with the tentative Coulomb excitation information [49], which influences the $S_{2 n}$ at ${ }^{62} \mathrm{Mn}$ and ${ }^{64} \mathrm{Mn}$. The inset shows the difference between the recalculated values (full line) and the original publication [48] (dashed line).

behavior [51], although the reported errors are relatively large. High-precision mass measurements are required to clarify the issue. To complete the picture, mean-square charge radii measurements for the $\mathrm{Cr}$ and $\mathrm{Fe}$ isotopes would be highly desirable.

\section{Duflo-Zuker analysis}

To conclude the discussion, the changes in mean-square charge radii of the $\mathrm{Mn}$ isotopes are compared to theoretical calculations using the Duflo-Zuker [52] formalism, shown in Fig. 9. It is based on the fact that radii are single-particle operators that depend on orbital occupancies and their sizes. A recent study [53] suggests that this formalism accounts for shell effects due to anomalously large orbits $\left(s_{1 / 2}, p_{3 / 2}\right.$, and $p_{1 / 2}$ in the neutron $s d$ and $p f$ shells, respectively), and leads to a reproduction of the experimental changes in root meansquare charge radii in $\mathrm{K}$ and $\mathrm{Ca}$.

The Duflo-Zuker expression consists of two terms,

$$
\begin{aligned}
\sqrt{\left\langle r_{\pi}^{2}\right\rangle}= & A^{1 / 3}\left[\rho_{0}-\frac{\zeta}{2} \frac{t}{A^{4 / 3}}-\frac{v}{2}\left(\frac{t}{A}\right)^{2}\right] e^{(g / A)} \\
& +\frac{\lambda}{A^{1 / 3}}\left[\frac{z\left(D_{\pi}-z\right)}{D_{\pi}^{2}} \frac{n\left(D_{v}-n\right)}{D_{v}^{2}}\right],
\end{aligned}
$$

where $t=N-Z$. Considering the terms (3a) and (3b) separately, the first term (3a) is derived by noting that $r_{\pi}$ is an isospin vector. Therefore, its square contains a scalar, a

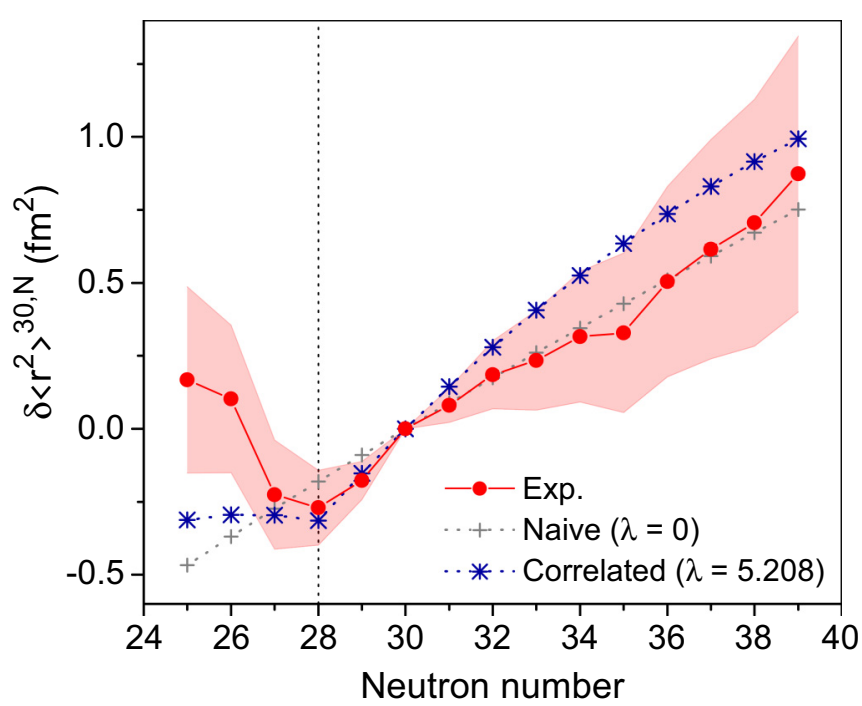

FIG. 9. Experimental mean-square charge radii of Mn compared to the naive (3a) and correlated $(3 a)+(3 b)$ predictions using the Duflo-Zuker expression.

vector and a tensor, associated with coefficients $\rho_{0}, \zeta$, and $v$, respectively. The expression for neutrons is obtained by reversing the sign of $t$, so the difference in neutron and proton charge radius, the neutron skin, is given by

$$
\Delta r_{\nu \pi}=\sqrt{\left\langle r_{\nu}^{2}\right\rangle}-\sqrt{\left\langle r_{\pi}^{2}\right\rangle}=\frac{\zeta t e^{g / A}}{A} .
$$

Here $e^{g / A}$ is a phenomenological correction factor which accounts for the larger radii in light nuclei. By a four-parameter fit to the known radii of isotopes with $A \leqslant 60, g=1.37$, $\rho_{0}=0.947 \mathrm{fm}, v=0.295 \mathrm{fm}$, and $\zeta=0.8 \mathrm{fm}$ are found, giving a rms deviation of $0.04 \mathrm{fm}$. Using this first term as a naive approach which only depends on $t$ and $A$, a smooth variation of the charge radii is obtained as shown in Fig. 9. The necessary shell effects are introduced by adding a second term (3b) which depends on the orbital occupancies of the extruder-intruder (EI) valence spaces, delimited by $N, Z=6,14,28,50, \ldots$ [54, Sec. IC]. The degeneracy of each valence shell is then given by $D_{\pi, \nu}=8,14,22, \ldots$ and the number of active particles is indicated by $n, z$. For the Mn isotopes this becomes $z=11$ and $D_{\pi}=14$ for the protons and $n=N-14, D_{v}=14$ for $N \leqslant 28$ or $n=N-28$ and $D_{v}=22$ for $N>28$. Note that this second term becomes zero at the extruder-intruder shell closures.

Once the second term (3b) is included in the fit, slightly varied fit parameters $\left(g=1.14, \rho_{0}=0.94 \mathrm{fm}, v=0.334\right.$ $\mathrm{fm}, \zeta=0.8 \mathrm{fm}$, and $\lambda=5.208$ ) are extracted and a good reproduction of the charge radii below $Z=30$ is obtained. This reduces the rms deviation to $0.02 \mathrm{fm}$.

The changes in mean-square charge radii obtained using the full Duflo-Zuker expression applied to the Mn isotopic chain (blue stars) are compared to the experimental values (red circles) in Fig. 9. Between $N=28$ and $N=35$, the agreement is good (taking into account the uncertainty on the experimental charge radii due to the uncertainty on $M$ and $F$, as indicated by the shaded area) and similar to $\mathrm{K}$ and $\mathrm{Ca}$, the 
strong increase in charge radii beyond $N=28$ is suggested to be associated with the large size of the (halo) neutron $p_{3 / 2}$ orbital [53]. Due to isovector polarizability [53], an increase of neutron radii causes larger charge (proton) radii as well. Nevertheless, above $N=35$ and below $N=28$ there are clear deviations between theory and experiment. This indicates that the large deformations, shown to be important for the Mn isotopes in this and previous work [19-22], are not fully taken into account in Eq. (3). Since in the $\mathrm{K}$ and $\mathrm{Ca}$ isotopes no similar large onset of deformation below $N=28$ is observed, the mean-square charge radii are well reproduced [53].

\section{CONCLUSION}

The ${ }^{51,53-64} \mathrm{Mn}$ isotopes have been studied in two collinear laser spectroscopy experiments at the COLLAPS beam line at ISOLDE, CERN. In this article, the isotope shifts and extracted mean-square charge radii were presented.

The electronic mass shift and field shift factors for the atomic $3 d^{5} 4 s^{2}{ }^{6} S_{5 / 2} \rightarrow 3 d^{5} 4 s 4 p{ }^{6} P_{3 / 2}$ and the ionic $3 d^{5} 4 s^{5} S_{2} \rightarrow 3 d^{5} 4 p^{5} P_{3}$ transitions have been calculated in the multiconfigurational Dirac-Fock framework. Because of the open $3 d$ electron shell, these calculations are particularly complex and relatively large uncertainties on $M$ and $F$ are assumed.

The nuclear structure along the $\mathrm{Mn}$ isotopic chain is discussed based on the available ground-state properties. A clear connection between the moments and charge radii is observed, pointing to a good $N=28$ shell closure and a rapid onset of deformation towards $N=40$. The variation of the two-neutron separation energies on the other hand is rather smooth, showing that these effects do not necessarily lead to discontinuities in the mass surface.
Furthermore, the evolution of the intrinsic shapes in the $\mathrm{Mn}$ isotopic chain was investigated in the Monte Carlo shell-model framework using the modified A3DA effective interaction. This suggests that the increase in charge radii from $N=35$ is not only due to a static increase in prolate deformation, but that also triaxiality and shape fluctuations are important.

Finally, the changes in mean-square charge radii were discussed using the Duflo-Zuker model. The sharp increase in charge radii beyond $N=28$, common to all isotopes studied in the region so far, has been related to the occupancy of the neutron (halo) orbitals. However, below $N=28$ and above $N=35$ the experimental values depart from the theoretical predictions, which is explained as due to deformation.

\section{ACKNOWLEDGMENTS}

We would like to thank the ISOLDE technical group for their support and assistance. H. H. thanks V. Manea for the interesting discussions and the insightful suggestions related to the separation energies. This work was supported by the Belgian Research Initiative on Exotic Nuclei (IAP Project P7/12), the FWO-Vlaanderen, GOA Grants No. 10/010 and No. 10/015 from KU Leuven, the German Ministry for Education and Research (BMBF) under Contracts No. 05P15RDICA and No. 05P15SJCIA, the Max-Planck Society, and the Science and Technology Facilities Council. This project has received funding through the European Union's Seventh Framework Programme for Research and Technological Development under Grant Agreements No. 262010 (ENSAR), No. 267194 (COFUND), and No. 289191 (LA3-NET).
[1] P. Campbell, I. D. Moore, and M. R. Pearson, Laser spectroscopy for nuclear structure physics, Prog. Part. Nucl. Phys. 86, 127 (2016).

[2] G. Neyens, Nuclear magnetic and quadrupole moments for nuclear structure research on exotic nuclei, Rep. Progr. Phys. 66, 633 (2003).

[3] K. Blaum, J. Dilling, and W. Nörtershäuser, Precision atomic physics techniques for nuclear physics with radioactive beams, Phys. Scr. T152, 014017 (2013).

[4] F. Touchard, P. Guimbal, S. Büttgenbach, R. Klapisch, M. D. S. Simon, J. Serre, C. Thibault, H. Duong, P. Juncar, S. Liberman, J. Pinard, and J. Vialle, Isotope shifts and hyperfine structure of ${ }^{38-47}$ K by laser spectroscopy, Phys. Lett. B 108, 169 (1982).

[5] C. W. P. Palmer, P. E. G. Baird, S. A. Blundell, J. R. Brandenberger, C. J. Foot, D. N. Stacey, and G. K. Woodgate, Laser spectroscopy of calcium isotopes, J. Phys. B 17, 2197 (1984).

[6] K. Blaum, W. Geithner, J. Lassen, P. Lievens, K. Marinova, and R. Neugart, Nuclear moments and charge radii of argon isotopes between the neutron-shell closures and, Nucl. Phys. A 799, 30 (2008).

[7] M. Avgoulea, Y. P. Gangrsky, K. P. Marinova, S. G. Zemlyanoi, S. Fritzsche, D. Iablonskyi, C. Barbieri, E. C. Simpson, P. D. Stevenson, J. Billowes, P. Campbell, B. Cheal, B. Tordoff,
M. L. Bissell, D. H. Forest, M. D. Gardner, G. Tungate, J. Huikari, A. Nieminen, H. Penttilä, and J. Äystö, Nuclear charge radii and electromagnetic moments of radioactive scandium isotopes and isomers, J. Phys. G 38, 025104 (2011).

[8] A. Huck, G. Klotz, A. Knipper, C. Miehé, C. Richard-Serre, G. Walter, A. Poves, H. L. Ravn, and G. Marguier, Beta decay of the new isotopes ${ }^{52} \mathrm{~K},{ }^{52} \mathrm{Ca}$, and ${ }^{52} \mathrm{Sc}$ : A test of the shell model far from stability, Phys. Rev. C 31, 2226 (1985).

[9] D. Steppenbeck, S. Takeuchi, N. Aoi, P. Doornenbal, M. Matsushita, H. Wang, H. Baba, N. Fukuda, S. Go, M. Honma, J. Lee, K. Matsui, S. Michimasa, T. Motobayashi, D. Nishimura, T. Otsuka, H. Sakurai, Y. Shiga, P.-A. Soderstrom, T. Sumikama, H. Suzuki, R. Taniuchi, Y. Utsuno, J. J. Valiente-Dobon, and K. Yoneda, Evidence for a new nuclear "magic number" from the level structure of ${ }^{54} \mathrm{Ca}$, Nature (London) 502, 207 (2013).

[10] F. Wienholtz, D. Beck, K. Blaum, C. Borgmann, M. Breitenfeldt, R. B. Cakirli, S. George, F. Herfurth, J. D. Holt, M. Kowalska, S. Kreim, D. Lunney, V. Manea, J. Menéndez, D. Neidherr, M. Rosenbusch, L. Schweikhard, A. Schwenk, J. Simonis, J. Stanja, R. N. Wolf, and K. Zuber, Masses of exotic calcium isotopes pin down nuclear forces, Nature (London) 498, 346 (2013).

[11] R. F. Garcia Ruiz, M. L. Bissell, K. Blaum, N. Frömmgen, M. Hammen, J. D. Holt, M. Kowalska, K. Kreim, J. Menéndez, R. 
Neugart, G. Neyens, W. Nörtershäuser, F. Nowacki, J. Papuga, A. Poves, A. Schwenk, J. Simonis, and D. T. Yordanov, Groundstate electromagnetic moments of calcium isotopes, Phys. Rev. C 91, 041304 (2015).

[12] R. F. Garcia Ruiz, M. L. Bissell, K. Blaum, A. Ekstrom, N. Frommgen, G. Hagen, M. Hammen, K. Hebeler, J. D. Holt, G. R. Jansen, M. Kowalska, K. Kreim, W. Nazarewicz, R. Neugart, G. Neyens, W. Nortershauser, T. Papenbrock, J. Papuga, A. Schwenk, J. Simonis, K. A. Wendt, and D. T. Yordanov, Unexpectedly large charge radii of neutron-rich calcium isotopes, Nat. Phys. 12, 594 (2016).

[13] J. Ljungvall, A. Görgen, A. Obertelli, W. Korten, E. Clément, G. de France, A. Bürger, J.-P. Delaroche, A. Dewald, A. Gadea, L. Gaudefroy, M. Girod, M. Hackstein, J. Libert, D. Mengoni, F. Nowacki, T. Pissulla, A. Poves, F. Recchia, M. Rejmund, W. Rother, E. Sahin, C. Schmitt, A. Shrivastava, K. Sieja, J. J. Valiente-Dobón, K. O. Zell, and M. Zielińska, Onset of collectivity in neutron-rich fe isotopes: Toward a new island of inversion? Phys. Rev. C 81, 061301 (2010).

[14] S. M. Lenzi, F. Nowacki, A. Poves, and K. Sieja, Island of inversion around ${ }^{64} \mathrm{Cr}$, Phys. Rev. C 82, 054301 (2010).

[15] Y. Utsuno, T. Otsuka, T. Glasmacher, T. Mizusaki, and M. Honma, Onset of intruder ground state in exotic $\mathrm{Na}$ isotopes and evolution of the $n=20$ shell gap, Phys. Rev. C 70, 044307 (2004).

[16] P. Himpe, G. Neyens, D. L. Balabanski, G. Bélier, J. M. Daugas, F. de Oliveira Santos, M. De Rydt, K. T. Flanagan, I. Matea, P. Morel, Y. E. Penionzhkevich, L. Perrot, N. A. Smirnova, C. Stodel, J. C. Thomas, N. Vermeulen, D. T. Yordanov, Y. Utsuno, and T. Otsuka, $g$ factor of the exotic isotope ${ }^{34} \mathrm{Al}$ : Probing the and shell gaps at the border of the "island of inversion," Phys. Lett. B 658, 203 (2008).

[17] G. Neyens, Multiparticle-multihole states in ${ }^{31} \mathrm{Mg}$ and ${ }^{33} \mathrm{Mg}$ : A critical evaluation, Phys. Rev. C 84, 064310 (2011).

[18] D. T. Yordanov, M. L. Bissell, K. Blaum, M. De Rydt, C. Geppert, M. Kowalska, J. Krämer, K. Kreim, A. Krieger, P. Lievens, T. Neff, R. Neugart, G. Neyens, W. Nörtershäuser, R. Sánchez, and P. Vingerhoets, Nuclear Charge Radii of ${ }^{21-32} \mathrm{Mg}$, Phys. Rev. Lett. 108, 042504 (2012).

[19] F. C. Charlwood, J. Billowes, P. Campbell, B. Cheal, T. Eronen, D. Forest, S. Fritzsche, M. Honma, A. Jokinen, I. D. Moore, H. Penttilä, R. Powis, A. Saastamoinen, G. Tungate, and J. Aystö, Ground state properties of manganese isotopes across the shell closure, Phys. Lett. B 690, 346 (2010).

[20] C. Babcock, H. Heylen, J. Billowes, M. L. Bissell, K. Blaum, P. Campbell, B. Cheal, R. F. Garcia Ruiz, C. Geppert, W. Gins, M. Kowalska, K. Kreim, S. M. Lenzi, I. D. Moore, R. Neugart, G. Neyens, W. Nörtershäuser, J. Papuga, and D. T. Yordanov, Evidence for increased neutron and proton excitations between ${ }^{51-63}$ Mn, Phys. Lett. B 750, 176 (2015).

[21] H. Heylen, C. Babcock, J. Billowes, M. L. Bissell, K. Blaum, P. Campbell, B. Cheal, R. F. Garcia Ruiz, C. Geppert, W. Gins, M. Kowalska, K. Kreim, S. M. Lenzi, I. D. Moore, R. Neugart, G. Neyens, W. Nörtershäuser, J. Papuga, and D. T. Yordanov, Spins and magnetic moments of ${ }^{58,60,62,64} \mathrm{Mn}$ ground states and isomers, Phys. Rev. C 92, 044311 (2015).

[22] C. Babcock, H. Heylen, M. Bissell, K. Blaum, P. Campbell, B. Cheal, D. Fedorov, R. Garcia Ruiz, W. Geithner, W. Gins, T. Goodacre, L. Grob, M. Kowalska, S. Lenzi, B. Maass, S. Malbrunot-Ettenauer, B. Marsh, R. Neugart, G. Neyens, W.
Nörtershäuser, T. Otsuka, R. Rossel, S. Rothe, R. Sánchez, Y. Tsunoda, C. Wraith, L. Xie, and X. F. Yang, Quadrupole moments of odd $-a^{53-63} \mathrm{Mn}$ : Onset of collectivity towards $N=$ 40, Phys. Lett. B 760, 387 (2016).

[23] K. Kreim, M. L. Bissell, J. Papuga, K. Blaum, M. De Rydt, R. G. Ruiz, S. Goriely, H. Heylen, M. Kowalska, R. Neugart, G. Neyens, W. Nörtershäuser, M. Rajabali, R. S. Alarcón, H. H. Stroke, and D. T. Yordanov, Nuclear charge radii of potassium isotopes beyond, Phys. Lett. B 731, 97 (2014).

[24] S. Rothe, T. D. Goodacre, D. Fedorov, V. Fedosseev, B. Marsh, P. Molkanov, R. Rossel, M. Seliverstov, M. Veinhard, and K. Wendt, Laser ion beam production at CERN-ISOLDE: New features-more possibilities, Nucl. Instr. Methods Phys. Res., Sec. B 376, 91 (2016).

[25] E. Mané, J. Billowes, K. Blaum, P. Campbell, B. Cheal, P. Delahaye, K. Flanagan, D. Forest, H. Franberg, C. Geppert, T. Giles, A. Jokinen, M. Kowalska, R. Neugart, G. Neyens, W. Nörtershäuser, I. Podadera, G. Tungate, P. Vingerhoets, and D. T. Yordanov, An ion cooler-buncher for high-sensitivity collinear laser spectroscopy at isolde, EPJ A 42, 503 (2009).

[26] A. Mueller, F. Buchinger, W. Klempt, E. Otten, R. Neugart, C. Ekström, and J. Heinemeier, Spins, moments, and charge radii of barium isotopes in the range ${ }^{122-146} \mathrm{Ba}$ determined by collinear fast-beam laser spectroscopy, Nucl. Phys. A 403, 234 (1983).

[27] W. Nörtershäuser, Recent developments in collinear laser spectroscopy at COLLAPS/ISOLDE, Hyperfine Interact. 198, 73 (2010).

[28] J. Papuga, M. L. Bissell, K. Kreim, C. Barbieri, K. Blaum, M. De Rydt, T. Duguet, R. F. Garcia Ruiz, H. Heylen, M. Kowalska, R. Neugart, G. Neyens, W. Nörtershäuser, M. M. Rajabali, R. Sánchez, N. Smirnova, V. Somà, and D. T. Yordanov, Shell structure of potassium isotopes deduced from their magnetic moments, Phys. Rev. C 90, 034321 (2014).

[29] B. Cheal, T. E. Cocolios, and S. Fritzsche, Laser spectroscopy of radioactive isotopes: Role and limitations of accurate isotopeshift calculations, Phys. Rev. A 86, 042501 (2012).

[30] P. Jönsson, G. Gaigalas, J. Bieroń, C. F. Fischer, and I. Grant, New version: GRASP2K relativistic atomic structure package, Comp. Phys. Commun. 184, 2197 (2013).

[31] S. Fritzsche, C. Fischer, and G. Gaigalas, RELCI: A program for relativistic configuration interaction calculations, Comp. Phys. Commun. 148, 103 (2002).

[32] C. Nazé, E. Gaidamauskas, G. Gaigalas, M. Godefroid, and P. Jönsson, RIS3: A program for relativistic isotope shift calculations, Comp. Phys. Commun. 184, 2187 (2013).

[33] S. Fritzsche, The RATIP program for relativistic calculations of atomic transition, ionization, and recombination properties, Comp. Phys. Commun. 183, 1525 (2012).

[34] W. King, Isotope Shifts in Atomic Spectra (Plenum, New York, 1984).

[35] G. Fricke and K. Heilig, Nuclear Charge Radii (Springer, Berlin, 2004).

[36] Y. P. Gangrsky, K. P. Marinova, S. G. Zemlyanoi, I. D. Moore, J. Billowes, P. Campbell, K. T. Flanagan, D. H. Forest, J. A. R. Griffith, J. Huikari, R. Moore, A. Nieminen, H. Thayer, G. Tungate, and J. Äystö, Nuclear charge radii of neutron deficient titanium isotopes $44 \mathrm{Ti}$ and $45 \mathrm{Ti}$, J. Phys. G 30, 1089 (2004).

[37] D. M. Benton, E. C. A. Cochrane, and J. A. R. Griffith, Optical isotope shifts in the iron atom, J. Phys. B 30, 5359 (1997). 
[38] A. Steudel, U. Triebe, and D. Wendlandt, Isotope shift in Ni I and changes in mean-square nuclear charge radii of the stable Ni isotopes, Z. Phys. A 296, 189 (1980).

[39] M. L. Bissell, T. Carette, K. T. Flanagan, P. Vingerhoets, J. Billowes, K. Blaum, B. Cheal, S. Fritzsche, M. Godefroid, M. Kowalska, J. Krämer, R. Neugart, G. Neyens, W. Nörtershäuser, and D. T. Yordanov, $\mathrm{Cu}$ charge radii reveal a weak sub-shell effect at $n=40$, Phys. Rev. C 93, 064318 (2016).

[40] M. L. Bissell, J. Papuga, H. Naïdja, K. Kreim, K. Blaum, M. De Rydt, R. F. Garcia Ruiz, H. Heylen, M. Kowalska, R. Neugart, G. Neyens, W. Nörtershäuser, F. Nowacki, M. M. Rajabali, R. Sanchez, K. Sieja, and D. T. Yordanov, Proton-Neutron Pairing Correlations in the Self-Conjugate Nucleus ${ }^{38} \mathrm{~K}$ Probed via a Direct Measurement of the Isomer Shift, Phys. Rev. Lett. 113, 052502 (2014).

[41] Y. Tsunoda, T. Otsuka, N. Shimizu, M. Honma, and Y. Utsuno, Novel shape evolution in exotic $\mathrm{Ni}$ isotopes and configurationdependent shell structure, Phys. Rev. C 89, 031301 (2014).

[42] N. Shimizu, T. Abe, Y. Tsunoda, Y. Utsuno, T. Yoshida, T. Mizusaki, M. Honma, and T. Otsuka, New-generation Monte Carlo shell model for the K computer era, Progr. Theor. Exp. Phys. 2012, 01A205 (2012).

[43] K. Sato, N. Hinohara, K. Yoshida, T. Nakatsukasa, M. Matsuo, and K. Matsuyanagi, Shape transition and fluctuations in neutron-rich $\mathrm{Cr}$ isotopes around $n=40$, Phys. Rev. C 86, 024316 (2012).

[44] F. C. Charlwood, K. Baczynska, J. Billowes, P. Campbell, B. Cheal, T. Eronen, D. H. Forest, A. Jokinen, T. Kessler, I. D. Moore, H. Penttilä, R. Powis, M. Rüffer, A. Saastamoinen, G. Tungate, and J. Äystö, Nuclear charge radii of molybdenum fission fragments, Phys. Lett. B 674, 23 (2009).

[45] R. B. Cakirli, R. F. Casten, and K. Blaum, Correlations of experimental isotope shifts with spectroscopic and mass observables, Phys. Rev. C 82, 061306 (2010).

[46] P. Van Isacker, D. D. Warner, and D. S. Brenner, Test of Wigner's Spin-Isospin Symmetry From Double Binding Energy Differences, Phys. Rev. Lett. 74, 4607 (1995).
[47] M. Wang, G. Audi, A. Wapstra, F. Kondev, M. MacCormick, $\mathrm{X}$. Xu, and B. Pfeiffer, The AME2012 atomic mass evaluation, Chin. Phys. C 36, 1603 (2012).

[48] S. Naimi, G. Audi, D. Beck, K. Blaum, C. Böhm, C. Borgmann, M. Breitenfeldt, S. George, F. Herfurth, A. Herlert, A. Kellerbauer, M. Kowalska, D. Lunney, E. Minaya Ramirez, D. Neidherr, M. Rosenbusch, L. Schweikhard, R. N. Wolf, and $\mathrm{K}$. Zuber, Surveying the $n=40$ island of inversion with new manganese masses, Phys. Rev. C 86, 014325 (2012).

[49] L. P. Gaffney, J. Walle, B. Bastin, V. Bildstein, A. Blazhev, N. Bree, J. Cederkäll, I. Darby, H. Witte, D. DiJulio, J. Diriken, V. N. Fedosseev, C. Fransen, R. Gernhäuser, A. Gustafsson, H. Hess, M. Huyse, N. Kesteloot, T. Kröll, R. Lutter, B. A. Marsh, P. Reiter, M. Seidlitz, P. Duppen, D. Voulot, N. Warr, F. Wenander, K. Wimmer, and K. Wrzosek-Lipska, Low-energy coulomb excitation of ${ }^{62} \mathrm{Fe}$ and ${ }^{62} \mathrm{Mn}$ following in-beam decay of ${ }^{62} \mathrm{Mn}$, EPJ A 51, 1 (2015).

[50] R. Fossion, C. D. Coster, J. García-Ramos, T. Werner, and K. Heyde, Nuclear binding energies global collective structure and local shell-model correlations, Nucl. Phys. A 697, 703 (2002).

[51] Z. Meisel, S. George, S. Ahn, D. Bazin, B. A. Brown, J. Browne, J. F. Carpino, H. Chung, R. H. Cyburt, A. Estradé, M. Famiano, A. Gade, C. Langer, M. Matoš, W. Mittig, F. Montes, D. J. Morrissey, J. Pereira, H. Schatz, J. Schatz, M. Scott, D. Shapira, K. Sieja, K. Smith, J. Stevens, W. Tan, O. Tarasov, S. Towers, K. Wimmer, J. R. Winkelbauer, J. Yurkon, and R. G. T. Zegers, Time-of-flight mass measurements of neutron-rich chromium isotopes up to $n=40$ and implications for the accreted neutron star crust, Phys. Rev. C 93, 035805 (2016).

[52] J. Duflo and A. P. Zuker, Mirror displacement energies and neutron skins, Phys. Rev. C 66, 051304 (2002).

[53] J. Bonnard, S. M. Lenzi, and A. P. Zuker, Neutron Skins and Halo Orbits in the $s d$ and $p f$ Shells, Phys. Rev. Lett. 116, 212501 (2016).

[54] E. Caurier, G. Martínez-Pinedo, F. Nowacki, A. Poves, and A. P. Zuker, The shell model as a unified view of nuclear structure, Rev. Mod. Phys. 77, 427 (2005). 